\title{
Enumeração e identificação de víbrios sacarose- positivos em amostras de lulas frescas comercializadas no município de Niterói-RJ
}

\section{(Enumeration and identification of sucrose-positive vibrios in samples of fresh squids commercialized at Niterói county-RJ)}

\author{
Francisco Carlos de Lima*, Luiz Antônio Trindade de Oliveira** e Rosana Rangel Duarte ${ }^{* * *}$
}

\begin{abstract}
Resumo
Foi efetuado um estudo sobre a enumeração e a identificação de víbrios sacarose-positivos em lulas frescas obtidas no comércio varejista do município de Niterói-RJ. No experimento, em 50 amostras de lulas, identificadas como pertencentes à espécie Dorytheutis brasiliensis Blainville, 1823, foram isoladas Unidades Formadoras de Colônias (UFC) sacarose-positivas em 28 (56\%) amostras. Destas, foram identificados o Vibrio alginolyticus e víbrios do grupo NAG (não aglutináveis). A média dos Números Mais Prováveis (NMP) dos víbrios totais (com exceção da amostra com NMP > 2400 bacts./g) foi 101,70 bacts./g. Do total de 29 isolamentos nas 28 amostras, o V. alginolyticus do grupo I de Heiberg obteve o maior percentual $(62,07 \%), \circ \mathrm{V}$. alginolyticus do grupo III apenas 10,30\% e os víbrios NAG com 27,63\%.
\end{abstract}

Palavras-chave: vibrios marinhos, Vibrio alginolyticus, vibrios NAG, vibrios sacarose-positivos.

\section{Introdução}

O gênero Vibrio engloba uma gama de espécies bastante semelhantes fenotipicamente (Johnson et al., 1971; Liston e Baross, 1973). Nos estudos sobre víbrios halofílicos na costa japonesa, Sakazaki et al. (1963) descreveram três biotipos, sendo que o biotipo II, o Vibrio alginolyticus, era também capaz de originar toxinfecção alimentar, além de ser o único a mostrar o fenômeno do "swarming" (crescimento abundante com espalhamento) sobre a superfície do agár com 2 a 7\% de NaCl (Sakazaki, 1968; Johnson et al., 1971). Dos organismos halofíicos, o V. alginolyticus é a espécie mais comumente isolada do ambiente marinho (Bockemhul e Triemer, 1974; Gjerde e Böe, 1981; Zebral, 1988; Chan, 1989). No Japão, Zen-Yoji et al. (1965) cons- tataram que o V. alginolyticus era responsável por 0,8\% dos casos de toxinfecções alimentares.

A maioria dos trabalhos sobre a patogenicidade do $\mathrm{V}$. alginolyticus reporta a predominância do organismo em lesões superficiais (Rubin e Tilton, 1975). Na Inglaterra, Ryan (1976) isolou o víbrio em três casos de lesões sépticas superficiais, sendo duas em feridas de extremidades e uma de secreção oriunda de otite externa. Pien et al. (1977), no Hawai, em oito casos estudados, isolaram a bactéria de três pacientes com otite externa e cinco a partir de lacerações e ferimentos superficiais, todos originados no ambiente marinho. Outros casos de infecções de ouvido pelo $\mathrm{V}$. alginolyticus foram assinalados por Olsen (1968) e Hansen et al. (1979), com os últimos identificando, na Bélgica, uma linhagem com características incomuns, como encapsulamento e imobilidade. Ainda em 1979, Schimidt et al., isolaram o organismo de secreção ocular em um caso de conjuntivite e Pezzlo et al., (1979) em um paciente com lesão ulcerativa na perna.

Os víbrios NAG (non-agglutinable) ou NVC (non-cholerae vibrio), assim designados por Chatterjee et al. (1970), não sofrem aglutinação pelo antisoro O/subgrupo I do V. cholerae (Aldova et al., 1968; Chatterjee et al., 1970; Muller, 1978), embora possuam reações similares às apresentadas por este. Os víbrios NAG são encontrados, com freqüência significativa, em pacientes com síndrome coleriforme (Aldova et al., 1968), podendo provocar diarréia grave semelhante à causada pelo biotipo El Tor do V. cholerae (Zafari et al., 1973). Dakin et al. (1975) cita um surto de gastrenterite envolvendo 64 pessoas, em que a presença de víbrios NAG foi detectada em alimentos de origem não marinha. Fearrington et al. (1974) e Prats et al. (1975) isolaram o microrganismo em dois casos distintos de septicemia seguida de encefalomielite.

*Prof. Adjunto - Dep. de Tecnologia de Alimentos/Fac. Vet. UFF

**Prof. Titular - Dep. de Tecnologia de Alimentos/Fac. Vet. UFF

***Médica Veterinária e Mestranda do Curso de Pós-Graduação em Higiene Processamento Tecnológico de Produtos de Origem Animal/Fac. Vet. UFF Rua Vital Brazil Filho, 64, Niterói, RJ/Brasil CEP: 24230-340 
O objetivo desta pesquisa é a identificação de víbrios sacarose-positivos potencialmente patogênicos, presentes no pescado comercializado no município de Niterói-RJ.

\section{Material e Métodos}

Foram utilizadas 50 amostras de lulas frescas, obtidas no mercado varejista do município de Niterói-RJ, no período de março a dezembro. As amostras, acondicionadas em isopor com gelo, foram levadas para o Laboratório de Controle Microbiológico de Produtos de Origem Animal da Faculdade de Veterinária da Universidade Federal Fluminense, onde após fragmentação e pesagem, foram homogeneizadas em liquidificador, por dois minutos em rotação média.

A enumeração dos víbrios sacarose-positivos foi feita de acordo com a metodologia prescrita pela International Commission on Specifications for Foods (ICMSF) de 1983, com as seguintes modificações:

a) Para a enumeração optou-se pelo meio GSTB (glucose-salt-teepol broth) (Akiyama et al., 1963, citado por Barros, 1977, p. 25) ao invés do caldo polimixina;

b) Na prova de Voges-Proskauer foi usada a temperatura de $35^{\circ}-37^{\circ} \mathrm{C} / 24-48$ horas, segundo MacFaddin (1985), ao invés de $27^{\circ}-29^{\circ} \mathrm{C} / 18$ horas;

c) Na formulação do GSTB usou-se o Tween 80 (Merck art. 822187) em substituição ao teepol. Ambas as substâncias têm a função de aumentar a tensão superficial do meio, dificultando a motilidade, o que evita a formação do "swarming" propiciando maior número de Unidades Formadoras de Colônias (UFC) isoladas.

Como teste complementar na identificação dos víbrios sacarose-positivos foi utilizada a Classificaçãó de Heiberg (1936), baseada na resposta à fermentação dos açúcares manose, sacarose e arabinose. Segundo esta classificação, o V. alginolyticus pode pertencer a dois grupos distintos: o I (manose +/ sacarose +/arabinose -) e o III (manose +/ sacarose + / arabinose + ); e os víbrios $N A G$, por sua vez aos grupos I (já citado), ao II (manose -/ sacarose +/ arabinose -) e ao $\vee$ (linhagens sacarose-negativas) (Chatterjee, 1974).

\section{Resultados}

Todas as amostras, quando inoculadas no meio GSTB, apresentaram crescimento. Em 35 amostras, equivalentes a um percentual de $70 \%$, foi observado o desenvolvimento

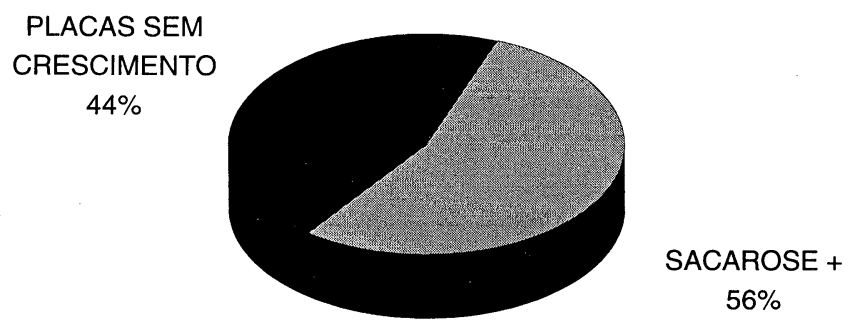

Fig. 1 - Percentual de frequência de bactérias sacarose positivas em 50 amostras de lulas.
Tabela 1 - Enumeração de víbrios totais, vibrios NAG e Vibrio alginolyticus, em amostras de lulas.

\begin{tabular}{|c|c|c|c|}
\hline № & $\begin{array}{l}\text { Vibrios Totais } \\
\text { NMP bacts./g }\end{array}$ & $\begin{array}{l}\text { Vibrios NAG } \\
\text { NMP bacts./g }\end{array}$ & $\begin{array}{l}\text { Vibrio alginolyticus } \\
\text { NMP bacts./g }\end{array}$ \\
\hline 01 & 15 & - & 15 \\
\hline 02 & 4 & - & 4 \\
\hline 03 & 15 & - & 15 \\
\hline 04 & 93 & - & 93 \\
\hline 05 & 43 & - & 43 \\
\hline 06 & 23 & 23 & - \\
\hline 07 & 460 & - & 460 \\
\hline 08 & 15 & - & 15 \\
\hline 09 & 4 & - & 4 \\
\hline 10 & 4 & 4 & - \\
\hline 11 & 4 & - & 4 \\
\hline 12 & 11 & - & 11 \\
\hline 13 & 3 & - & 3 \\
\hline 14 & 23 & - & 23 \\
\hline 15 & 7 & - & 7 \\
\hline 16 & 150 & - & 150 \\
\hline 17 & 21 & - & 21 \\
\hline 18 & 15 & - & 15 \\
\hline 19 & 93 & - & 93 \\
\hline 20 & 11 & 11 & - \\
\hline 21 & 21 & 21 & - \\
\hline 22 & 75 & - & 75 \\
\hline 23 & 460 & - & 460 \\
\hline 24 & 39 & 39 & - \\
\hline 25 & 9 & 9 & - \\
\hline 26 & 1100 & 1100 & - \\
\hline 27 & 28 & 28 & - \\
\hline 28 & $>2400$ & - & $>2400$ \\
\hline
\end{tabular}

Tabela 2 - Perfil bioquímico do Vibrio alginolyticus, e vibrios NAG isolados de amostras de lulas

\begin{tabular}{|c|c|c|c|}
\hline \multicolumn{2}{|c|}{ Provas bioquímicas } & Vibrio alginolyticus & Vibrios NAG \\
\hline \multicolumn{2}{|c|}{ Citocromo-oxidase } & + & + \\
\hline \multicolumn{2}{|c|}{$\begin{array}{c}\text { Citocromo-oxidase } \\
\text { VP }\end{array}$} & + & $6-/ 2+$ \\
\hline \multicolumn{2}{|c|}{ Crescimento a $42^{\circ} \mathrm{C}$} & + & + \\
\hline \multicolumn{2}{|c|}{$\begin{array}{c}\text { Lisina } \\
\text { Arginina }\end{array}$} & + & + \\
\hline Argin & & $16-/ 4+$ & - \\
\hline \multirow[t]{4}{*}{ Halofilismo } & $0 \%$ & - & $7-/ 1+$ \\
\hline & $6 \%$ & + & + \\
\hline & $8 \%$ & + & + \\
\hline & $10 \%$ & + & $7+/ 1-$ \\
\hline \multicolumn{2}{|c|}{ Glicose } & + & + \\
\hline \multirow{2}{*}{\multicolumn{2}{|c|}{$\begin{array}{l}\text { Sacarose } \\
\text { Manitol }\end{array}$}} & + & + \\
\hline & & + & $7+/ 1-$ \\
\hline
\end{tabular}

(+) amostra positiva

$(-)$ amostra negativa

máximo (crescimento nos três tubos das três séries) e em 47 amostras, nas diluições de $10^{-1}$ e $10^{-2}$.

Na semeadura em agár TCBS (tiossulfato-citrato-sais biliares-sacarose), 28 amostras apresentaram formação de UFCs amarelas (sacarose-positivas), como pode ser observado na Fig. 1. Estas unidades eram de tamanhos variados com tendência a grandes. 
Tabela 3 - Diferenciação do Vibrio alginolyticus dos vibrios NAG, em amostras de lulas

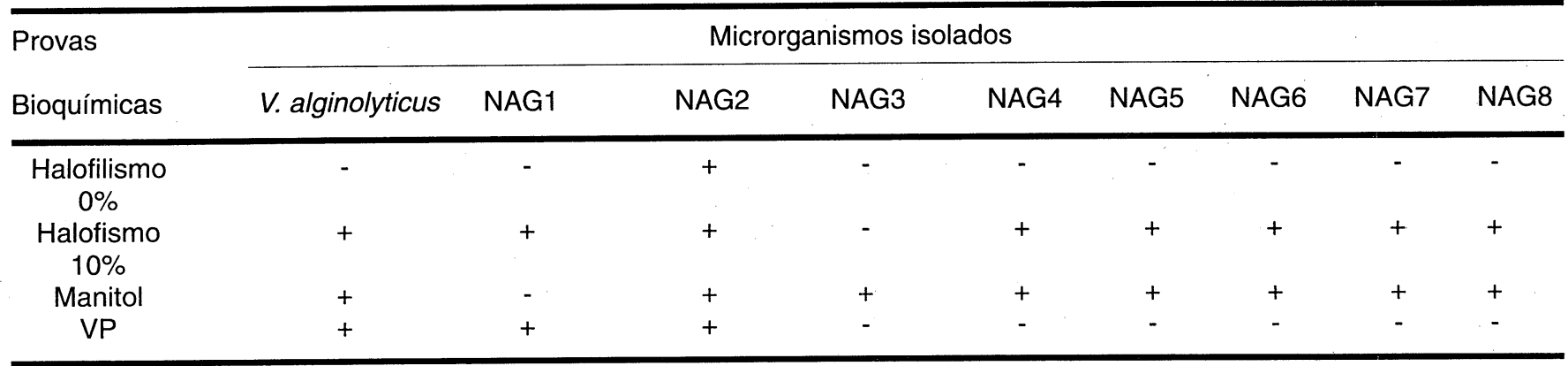

Tabela 4 - Distribuição das 29 amostras de lulas segundo a Prova de Heiberg

\begin{tabular}{lcccrr}
\hline \multicolumn{1}{c}{ Espécies } & Sacarose & Manose & Arabinose & No de isolamentos & \% \\
\hline Vibrio alginolyticus (I) & + & + & - & 18 & 62,07 \\
Vibrio alginolyticus (III) & + & + & + & 03 & 10,30 \\
Vibrios NAG (II) & + & - & - & 08 & 27,63 \\
\hline TOTAL & & & & 29 & 100,00 \\
\hline
\end{tabular}

O exame morfológico evidenciou que todas as UFCs eram formadas por bactérias Gram negativas, pleomórficas, porém com número significativo de organismos em forma de bastões curvos.

Pelas características tintoriais e morfológicas, os microrganismos isolados foram considerados como pertencentes ao grupo Vibrio. As amostras, com exceção de uma com NMP (Número Mais Provável) máximo (> 2.400 bacts./g) (Tabela 1), apresentaram o NMP médio de 101,70 bacts./g, e 23 (85,18\%) com NMP abaixo do índice médio.

No agár motilidade todas as bactérias mostraram-se móveis e apresentaram crescimento no caldo e no agár tripticase soja. Nas semeaduras em TSI, houve acidificação tanto na base quando no bisel, tornando o meio totalmente amarelo.

Os testes bioquímicos evidenciaram que das 28 amostras com UFCs sacarose-positivas no meio TCBS, 20 $(71,40 \%)$ continham vibrios que apresentaram um perfil bioquímico semelhante ao do $\mathrm{V}$. alginolyticus (Tabela 2). Os oito restantes, correspondendo a $28,60 \%$, embora possuidores de respostas bioquímicas bastante semelhantes àquelas do V. alginolyticus (Tabela 2), foram diferenciados através de testes associados (Tabela 3) e identificados pela prova de Heiberg (Tabela 4) como pertencentes ao grupo NAG.

Segundo a Classificação de Heiberg, das 20 amostras positivas para o V. alginolyticus, uma desenvolveu UFCs com resposta distinta em relação à utilização da arabinose. Destes 21 isolamentos do $\mathrm{V}$. alginolyticus, 18 pertenciam ao grupo I e três ao grupo III de Heiberg. Os organismos do grupo. NAG, identificados pelo teste de Heiberg, foram diferenciados dos grupos aos quais pertenciam $\mathrm{OV}$. alginolyticus pela não utilização da manose (Tabela 4), o que os caracterizavam como pertencentes ao grupo II de Heiberg.

\section{Discussão}

Os cefalópodes, e em especial, as lulas, foram substratos raramente utilizados em estudos microbiológicos até a presente data, acarretando uma limitação muito grande na comparação dos parâmetros analisados.

A maioria das pesquisas envolvendo os víbrios sacarosepositivos tomou amostras coletadas no ambiente marinho, enquanto neste trabalho as amostras foram coletadas em bancas de revenda ao consumidor, estando sujeitas a vários fatores que poderiam acarretar modificações em sua carga microbiana.

Em relação ao meio TCBS, não foi registrado nenhum crescimento anormal das UFCs sacarose-positivas no tempo de incubação preconizado. A formação de "swarming" só foi verificada nas UFCs do V. alginolyticus, em placas incubadas em um tempo mínimo de 48 horas. A substituição do "teepol" pelo "tween 80", no meio GSTB, pareceu não modificar a característica desejada de inibir a formação do "swarming".

O V. alginolyticus pode ser identificado por meio de poucas características bioquímicas e morfológicas, sendo facilmente diferenciável de outros víbrios (Pezzlo et al., 1979). No entanto, várias pesquisas têm mostrado que algumas provas podem apresentar respostas incomuns que dificultam a sua identificação. A literatura cita linhagens sacarose-negativas do V. alginolyticus (Sakazaki et al., 1963; Zen-Yoji et al. 1965), além do teste VP negativo (Zen-Yoji et al., 1965; Pien et al., 1977; Schimidt et al., 1979) e sem crescimento em meio salino a 10\% (Zen-Yoji et al., 1965) e a 8\% (Schimidt et al., 1979).

As 20 amostras de V. alginolyticus isoladas em lulas apresentaram o padrão normal nas três provas citadas, ou seja, positivas para sacarose, VP e halofilismo a 10\%, não tendo 
Francisco Carlos de Lima, Luiz Antônio Trindade de Oliveira e Rosana Rangel Duarte

sido observadas cepas imóveis como relatado por Hansen et al: (1979) e sem crescimento a $42^{\circ} \mathrm{C}$ como as citadas por Kristensen (1974) e Ryan (1976). Os testes básicos aplicados a estas amostras apresentaram os resultados esperados para a caracterização da espécie, exceção feita somente à prova da arginina, normalmente negativa (Kampelmacher e Jansen, 1972; Zen-Yoji et al., 1973; Kristensen, 1974; Ryan, 1976; Pezzlo et al., 1979; Blake et al. 1980), mas que apresentou resultado positivo em quatro das amostras analisadas. Este resultado indica uma freqüência bastante alta em relação ao obtido por Baumann et al. (1973) que encontraram, em 30 amostras do V. alginolyticus, apenas uma arginina positiva.

A posição do V. alginolyticus dentro da classificação de Heiberg é dada, principalmente, pela prova de fermentação de arabinose, já que a da manose pareceu ser positiva em todos os trabalhos consultados e a da sacarose, em razão da rara incidência de negativos, não é significativa para a criação de um novo grupo. Na maioria das pesquisas, as amostras do $\mathrm{V}$. alginolyticus são arabinose-negativas, o que as situam no grupo I de Heiberg.

Neste trabalho foram verificadas linhagens do $V$. alginolyticus fermentadoras da arabinose, semelhantes às relatadas por Kampelmacher e Jansen (1972) é Chatterjee (1974), que isolaram duas destas linhagens em dez e 16 em 43 amostras examinadas, respectivamente. Dos 21 isolados do V. alginolyticus obtidos das amostras de lulas, três foram arabinose-positivos, sendo incluídos no grupo III de Heiberg e os 18 arabinose-negativos, foram considerados pertencentes ao grupo I.

Em relação ao víbrio NAG e à sua posição na classificação de Heiberg, Bhattacharji e Bose (1964) verificaram a presença destes víbrios na água do mar, principalmente os dos grupos I e II. Sakazaki et al. (1967) encontraram 88,10\% das amostras com respostas inerentes ao grupo $1,9,30 \%$ ao grupo II e apenas 2,60\% ao grupo V. Víbrios NAG dos grupos I e || foram também isolados por Dakin et al. (1974), Muller (1977), Muller (1978) e Smith Jr. (1979), sendo que Muller (1977) observou um predomínio três vezes maior de víbrios do grupo II.

O isolamento de oito amostras de víbrios NAG do grupo II, em lulas, não oferece uma base sólida para uma discussão efetiva embora outros autores como Aldova et al. (1968) e Chatterjee et al. (1970) tenham encontrado apenas víbrios NAG do grupo II em suas pesquisas.

O percentual de $27,63 \%$ de víbrios NAG isolados, do total de organismos sacarose-positivos está de acordo com os valores determinados por Muller (1977).e Muller (1978) que são de 22 e 33\%, respectivamente, na água do mar.

\section{Abstract \\ The enumeration and identification of sucrose- positive vibrios in fresh squids commercialized at Niterói county RJ}

From 50 samples of squids of the species Dorytheutis brasiliensis Blainville, 1823, Colonies Formed Units (CFUs) sucrose-positives Vibrio alginolyticus and NAG vibrios (non- agglutinable) were isolated. The average of the Most Probabble Number of total vibrios (except the sample with MPN > 2400 bacts./g) was 101,70 bacts./g. From 28 samples, 29 isolations were carried out and the $V$. alginolyticus group I of Heiberg's Classification showed the greatest percentage (62,07\%). The V. alginolyticus group III showed only $10,30 \%$ and NAG vibrios $27,63 \%$.

Key words: marine vibrios, Vibrio alginolyricus, NAG vibrios, sucrosepositive vibrios.

\section{Referências}

ALDOVA, E., LAZNICKOVA, K., STEPANKOVA, E. LIETAVA, J. Isolation of Nonnagglutinable Vibrios from an Enteritis Outbreak in Czechoslovakia. Journal of Infectious Diseases, v. 118, n. 1, p. 25-31, 1968.

BARROS, G.C. Vibrio parahaemolyticus: Isolamento e Identificação em Crustáceos e Moluscos da Baia de Sepetiba. Niterói, 1977. 70p. Dissertação de Mestrado em Higiene e Processamento de Produtos de Origem Animal - Universidade Federal Fluminense.

BAUMANN, P., BAUMANN, L., REICHELT, J.L. Taxonomy of Marine Bacteria; Beneckea parahaemolytica and Beneckea alginolytica. Journal of Bacteriology, v. 113, n. 3, p. 1144-1155, 1973.

BHATTACHARJI, L.M., BOSE, B. Field and Laboratory Studies on the Transformation of the Vibrio cholerae in the Maintenance of Cholera Endemicity: a Preliminary Report. Indian Journal of Medical Research, v. 52, p. 777-783, 1964.

BLAKE, P.A. WEAVER, R.E., HOLLIS, D.G. Diseases of Humans (other than cholera) Caused by Vibrios. Annual Review of Microbiology, v. 34, p. 341-367, 1980.

BOCKEMUHL, J., TRIEMER, A. Ecology and Epidemiology of Vibrio parahaemolyticus on the Coast of Togo. Bulletin of the World Health Organization, v. 51, n. 4, p. 353-360, 1974.

CHAN, K.-Y., WOO, M.L., LAM, L.Y., FRENCH, G.L. Vibrio parahaemolyticus and other Halophilic Vibrios Associated with Seafood in Hong Kong. Journal of Applied Bacteriology, v. 66, n. 1, p. 57-64, 1989.

CHATTERJEE, B.D. Present Status of Heiberg Groups for Classifyng Choleralike Organisms. Indian Journal of Medical Research, v. 62, p. 479-483, 1974

CHATTERJEE, B.D., GORBACH, S.L., NEOGY, K.N. Characteristics of Noncholera Vibrios Isolated from Patients with Diarrhoea. Journal of Medical Microbiology, v. 3, n. 4, p. 677-682, 1970.

DAKIN, W.P.H., HOWELL, D.J., SUTTON, R.G.A., D'KEEFE, M.F., THOMAS, P. Gastroenteritis due to Non-agglutinable (non-cholera) Vibrios. Medical Journal of Australia, v. 2, n. 13, p. 487-490, 1974

FEARRINGTON, E.L., RAND Jr., C.H., MEWBORN, A. WILKERSON, J. Noncholera Vibrio Septicemia and Meningoencephalitis. Annals of Internal Medicine, v. 81, n. 3, p. 401, 1974

GJERDE, J., BÖE, B. Isolation and Characterization of Vibrio alginolyticus and Vibrio parahaemolyticus from the Norwegian Coastal Environment. Acta Veterinaria Scandinavica, v. 22, p. 331-343, 1981

HANSEN, W., CROKAERT, F., YOURASSOWSKY, E. Two strains of Vibrio species with Unusual Biochemical Features Isolated from Ear Tracts. Journal of Clinical Microbiology, v. 9, n. 1, p. 152-153, 1979.

HEIBERG, B. The Biochemical Reactions of Vibrios. Journal of Hygiene, London, v. 36 p. 114-117, 1936

INTERNATIONAL COMMISSION ON MICROBIOLOGICAL SPECIFICATIONS FOR FOODS. Microorganismos de los alimentos. 2 ed. Zaragoza, Editorial Acribia, 1983. v. 1: Tecnicas de analises microbiologico. 431p

JOHNSON, H.C., BAROSS, J.A., LISTON, J. Vibrio parahaemolyticus and its Importance in Seafood Hygiene. Journal of the American Veterinary Medical Association. v. 159, n. 11, p. 1470-1473, 1971

KAMPELMACHER, E.H., JANSEN, L.M. VAN N. A Survey of the occurrence of Vibrio parahaemolyticus and $V$. alginolyticus, on Mussels and Oysters 
and in Estuarine Waters in the Netherlands: Journal of Applied Bacteriology, v. 35, n. 3, p. 431-438, 1972

KRISTENSEN, K.K. The Occurrence of Vibrio parahaemolyticus and Vibrio alginolyticus in the Sound. Nordisk Veterinaermedicin, v. 26, n. 3/4, p. 188-196, 1974

LISTON, J., BAROSS, J. Distribution of Vibrio parahaemolyticus in the Natural Environment. Journal of Milk and Food Technology, v. 36, n. 2, p. 113-117, 1973

MACFADDIN, J.F. Pruebas Bioquimicas parala ldentificacion de las Bacterias de Importancia Clinica. Buenos Aires: Panamericana, 1985. 301p.

MULLER, G. Befunde an Nicht-agglutinierenden, Cholera-ähnlichen Vibrionen (NAG's) in Abwasser, Flubwasser und Meerwasser. Zentralblatt fur Bakteriologie, Mikrobiologie und Hygiene, Abt. I. Orig. B, v. 165, n. 5/6, p. $487-497,1977$.

MULLER, H.E. Vorkomen und Ökologie non NAG-vibrionen in Oberflächengewässern. Zentralblatt fur Bakteriologie, Mikrobiologie und Hygiene, Abt. I. Origi. B, v. 167, n. 3, p. 272-284, 1978.

OLSEN, H., Vibrio parahaemolyticus Isolated from Discharge from the Ear in Two Patients Exposed to Sea Water. Acta Pathologica et Microbiologica Scandinavica. Section B, v. 86, n. 4, p. 247-248, 1978.

PEZZLO, M., VALTER, P.J., BURNS, M.J. Wound Infection Associated with Vibrio alginolyticus. American Journal of Clinical Pathology, v. 71, n. 4, p. 476-478, 1979

PIEN, F., LEE, K., HIGA, H. Vibrio alginolyticus Infections in Hawaï. Journal of Clinical Microbiology, v. 5, n. 6, p. 670-672, 1977

PRATS, G., MIRELIS, B., PERICAS, R. Non-cholera Vibrio Septicemia and Meningo-encephalitis. Annals of Internal Medicine, v. 82, n. 6, p. 848849,1975

RUBIN, S.J., TILTON, R.C. Isolation of Vibrio alginolyticus from Wound Infection. Journal of Clinical Microbiology, v. 2, n. 12, p. 1556-1558, 1975
RYAN, W.J. Marine Vibrios Associated with Superficial Septic Lesions. Journal of Clinical Pathology, v. 29, n. 11, p. 1114-1115, 1976.

SAKAZAKI, R. Proposal of Vibrio alginolyticus for the Biotype 2 of Vibrio parahaemolyticus. Japanese Journal of Medical Science and Biology, v. 21, n. 6, p. 359-362, 1968

SAKAZAKI, R., GOMEZ, C.Z., Sebald, M. Taxonomical Studies of the Socalled NAG Vibrios. Japanese Journal of Medical Science and Biology,

v. 20, p. 265-280, 1967.

SAKAZAKI, R., IWANAMI, S., FUKUMI, H. Studies on the Enteropathogenic, Facultatively Halophilic Bacteria, Vibrio parahaemolyticus. I. Morphological, Cultural and Biochemical Properties and its Taxonomical Positions. Japanese Journal of Medical Science and Biology, v. 16, p. $161-188,1963$

SCHIMIDT, U., CHMEL, H., COBBS, C. Vibrio alginolyticus Infections in Humans. Journal of Clinical Microbiology, v. 10, n. 5, p. 666-668, 1979

SMITH Jr., H.L. Serotyping of Non-cholera Vibrios. Journal of Clinical Microbiology, v. 10, n. 1, p. 85-90, 1979

ZAFARI, Y., RAHAMANZADEH, S., ZARIFI, A.Z. ZAFARI, Y. Diarrhoea Caused by Non-agglutinable Vibrio cholerae (non-cholera vibrio). Lancet, v. 2 , p. $429-430,1973$.

ZEBRAL, A.A. Isolamento e Caracterização de Vibrio parahaemolyticus, outros Vibriões e Bactérias Afins, de Mexilhões da Baia de Guanabara. Ciências Médicas, v. 7, n. 1/2, p. 21-28, 1988.

ZEN-YOJI, H., LECLAIR, R.A., OHTA, K. MONTAGUE, T.S. Comparison of Vibrio parahaemolyticus Cultures Isolated in United States with those Isolated in Japan. Journal of Infectious Diseases, v. 127, n. 3, p. 237$241,1973$.

ZEN-YOJI, H., SAKAI, S., TERAYAMA, T. KUDO, Y. ITO, T., BENOKI, M., NAGASAKI, M. Epedemiology, Enteropathogenicity and Classification of Vibrio parahaemolyticus. Journai of Infectious Diseases, v. 115, n. 5 , p. 436-444, 1965. 